\title{
Laparoscopic specimen retrieval bags in gyn surgery: Expert guidance on selection
}

\author{
For MIGS procedures, understanding the features of specimen retrieval \\ systems (which vary widely), as well as the pathology's characteristics, \\ is essential to surgical decision making
}

Tiffany Sia, MD, and Hye-Chun Hur, MD, MPH

\section{IN THIS} ARTICLE

Pathology determinants page 37

\section{Laparoscopic bag features page 38}

Bag shape, taper

page 41
 he use of minimally invasive gynecologic surgery (MIGS) has grown rapidly over the past 20 years. MIGS, which includes vaginal hysterectomy and laparoscopic hysterectomy, is safe and has fewer complications and a more rapid recovery period than open abdominal surgery. ${ }^{1,2}$ In 2005, the role of MIGS was expanded further when the US Food and Drug Administration (FDA) approved robot-assisted surgery for the performance of gynecologic procedures. ${ }^{3}$ As knowledge and experience in the safe performance of MIGS progresses, the rates for MIGS procedures have skyrocketed and continue to grow. Between 2007 and 2010, laparoscopic

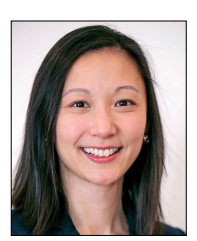

Dr. Sia is a Resident in Obstetrics and Gynecology, Columbia University College of Physicians and Surgeons, New York, New York.

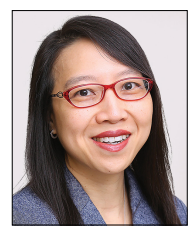

Dr. Hur is an Assistant Professor of Obstetrics and Gynecology, Columbia University Irving Medical Center and New York Presbyterian Hospital.

The authors report no financial relationships relevant to this article.

doi: 10.12788 /obgm .0042 hysterectomy rates rose from $23.5 \%$ to $30.5 \%$, while robot-assisted laparoscopic hysterectomy rates increased from $0.5 \%$ to $9.5 \%$, representing $40 \%$ of all hysterectomies. ${ }^{4}$ Due to the benefits of minimally invasive surgery over open abdominal surgery, patient and physician preference for minimally invasive procedures has grown significantly in popularity.,

Because incisions are small in minimally invasive surgery, surgeons have been challenged with removing large specimens through incisions that are much smaller than the presenting pathology. One approach is to use a specimen retrieval bag for specimen extraction. Once the dissection is completed, the specimen is placed within the retrieval bag for removal, thus minimizing exposure of the specimen and its contents to the abdominopelvic cavity and incision.

The use of specimen retrieval devices has been advocated to prevent infection, avoid spillage into the peritoneal cavity, and minimize the risk of port-site metastases in cases of potentially cancerous specimens. Devices include affordable and readily available products, such as nonpowdered gloves, and commercially produced bags. ${ }^{6}$

While the use of specimen containment systems for tissue extraction has been well described in gynecology, the available systems vary widely in construction, size, durability, and shape, potentially leading to confusion 
and suboptimal bag selection during surgery. ${ }^{7}$ In this article, we review the most common laparoscopic bags available in the United States, provide an overview of bag characteristics, offer practice guidelines for bag selection, and review bag terminology to highlight important concepts for bag selection.

\section{Controversy spurs change}

In April 2014, the FDA warned against the use of power morcellation for specimen removal during minimally invasive surgery, citing a prevalence of 1 in 352 unsuspected uterine sarcomas and 1 in 498 unsuspected uterine leiomyosarcomas among women undergoing hysterectomy or myomectomy for presumed benign leiomyoma. ${ }^{8}$ Since then, the risk of occult uterine sarcomas, including leiomyosarcoma, in women undergoing surgery for benign gynecologic indications has been determined to be much lower.

Nonetheless, the clinical importance of contained specimen removal was clearly highlighted and the role of specimen retrieval bags soared to the forefront. Open power morcellation is no longer commonly practiced, and national societies such as the American Association of Gynecologic Laparoscopists (AAGL), the Society of Gynecologic Oncology (SGO), and the American College of Obstetricians and Gynecologists (ACOG) recommend that containment systems be used for safer specimen retrieval during gynecologic surgery. ${ }^{9-11}$ After the specimen is placed inside the containment system (typically a specimen bag), the surgeon may deliver the bag through a vaginal colpotomy or through a slightly extended laparoscopic incision to remove bulky specimens using cold-cutting extraction techniques. ${ }^{12-15}$

\section{Know the pathology's characteristics}

In most cases, based on imaging studies and physical examination, surgeons have a good idea of what to expect before proceeding with surgery. The 2 most common characteristics used for surgical planning are the specimen size (dimensions) and the tissue type (solid, cystic, soft tissue, or mixed). The mass size can range from less than $1 \mathrm{~cm}$ to larger than a 20-week sized fibroid uterus. Assessing the specimen in 3 dimensions is important. Tissue type also is a consideration, as soft and squishy masses, such as ovarian cysts, are easier to deflate and manipulate within the bag compared with solid or calcified tumors, such as a large fibroid uterus or a large dermoid with solid components.

Specimen shape also is a critical determinant for bag selection. Most specimen retrieval bags are tapered to varying degrees, and some have an irregular shape. Long tubular structures, such as fallopian tubes that are composed of soft tissue, fit easily into most bags regardless of bag shape or extent of bag taper, whereas the round shape of a bulky myoma may render certain bags ineffective even if the bag's entrance accommodates the greatest diameter of the myoma. Often, a round mass will not fully fit into a bag because there is a poor fit between the mass's shape and the bag's shape and taper. (We discuss the concept of a poor "fit" below.) Knowing the pathology before starting a procedure can help optimize bag selection, streamline operative flow, and reduce waste.

\section{Overview of laparoscopic bag characteristics and clinical applications}

The TABLE (pages 38-39) lists the most common laparoscopic bags available for purchase in the United States. Details include the trocar size, manufacturer, product name, mouth 
SURGICAL technique

Laparoscopic specimen retrieval bags in gyn surgery: Expert guidance on selection

TABLE Features of common laparoscopic retrieval bags available in the United States

\begin{tabular}{|c|c|c|c|c|c|c|c|c|}
\hline $\begin{array}{l}\text { Trocar } \\
\text { size }\end{array}$ & $\begin{array}{c}\text { Product } \\
\text { name }\end{array}$ & $\begin{array}{c}\text { Mouth } \\
\text { diameter, } \\
\text { width X } \\
\text { length, cm }\end{array}$ & $\begin{array}{c}\text { Bag } \\
\text { height, } \\
\text { cm }\end{array}$ & $\begin{array}{c}\text { Volume, } \\
\text { mL }\end{array}$ & $\begin{array}{c}\text { Bag } \\
\text { taper }\end{array}$ & Material & $\begin{array}{c}\text { Clinical } \\
\text { application }\end{array}$ & Bag shape \\
\hline $5 \mathrm{~mm}$ & $\begin{array}{l}\text { TissueBag } \\
\text { Premium 5mm } \\
\text { (A.M.I.) }\end{array}$ & $4.6 \times 6.0$ & 17.0 & 100 & Minimal taper & Polyurethane & Salpingectomy & \\
\hline \multirow[t]{2}{*}{$8 \mathrm{~mm}$} & $\begin{array}{l}\text { Anchor TRS- } \\
\text { ROBO-8 } \\
\text { (ConMed) }\end{array}$ & $4.0 \times 6.6$ & 14.0 & 125 & Gradual taper & Ripstop nylon & $\begin{array}{c}\text { Small fibroid, } \\
\text { solid adnexal } \\
\text { mass }\end{array}$ & 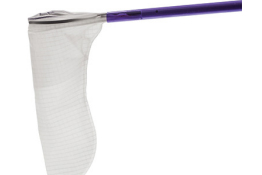 \\
\hline & $\begin{array}{c}\text { Inzii 5a } \\
\text { (Applied Medical) }\end{array}$ & $4.4 \times 8.0$ & 19.0 & 180 & Gradual taper & Polyurethane & Salpingectomy & \\
\hline \multirow[t]{6}{*}{$10 \mathrm{~mm}$} & $\begin{array}{c}\text { TissueBag } \\
\text { Premium 10mm } \\
\text { (A.M.I.) }\end{array}$ & $6.0 \times 8.0$ & 18.5 & 210 & Gradual taper & Polyurethane & $\begin{array}{l}\text { Oophorectomy, } \\
\text { simple cysts }\end{array}$ & $=$ \\
\hline & $\begin{array}{l}\text { Endo Catch } \\
\text { Gold } \\
\text { (Covidien/ } \\
\text { Medtronic) }\end{array}$ & $6.3 \times 7.1$ & 15.0 & 220 & Gradual taper & Polyurethane & $\begin{array}{l}\text { Oophorectomy, } \\
\text { simple cysts }\end{array}$ & $\simeq$ \\
\hline & $\begin{array}{l}\text { Endopouch } \\
\text { Retriever } \\
\text { (Ethicon) }\end{array}$ & $5.4 \times 5.8$ & 15.0 & 224 & Gradual taper & Polyurethane & $\begin{array}{l}\text { 0ophorectomy, } \\
\text { simple cysts }\end{array}$ & OEtnicon, Inc \\
\hline & $\begin{array}{c}\text { Inzii } 10 \\
\text { (Applied Medical) }\end{array}$ & $5.2 \times 7.8$ & 11.5 & 225 & Gradual taper & Polyurethane & $\begin{array}{l}\text { Oophorectomy, } \\
\text { simple cysts }\end{array}$ & \\
\hline & $\begin{array}{l}\text { Anchor TRS100SB2 } \\
\text { (ConMed) }\end{array}$ & $4.9 \times 8.1$ & 14.0 & 235 & Gradual taper & Ripstop nylon & $\begin{array}{l}\text { Dermoids, } \\
\text { fibroids }\end{array}$ & \\
\hline & $\begin{array}{l}\text { ReliaCatch } \\
10 \mathrm{~mm} \\
\text { (Covidien/ } \\
\text { Medtronic) }\end{array}$ & $6.4 \times 7.7$ & 16.6 & 275 & Gradual taper & Ripstop nylon & $\begin{array}{l}\text { Dermoids, } \\
\text { fibroids }\end{array}$ & \\
\hline
\end{tabular}




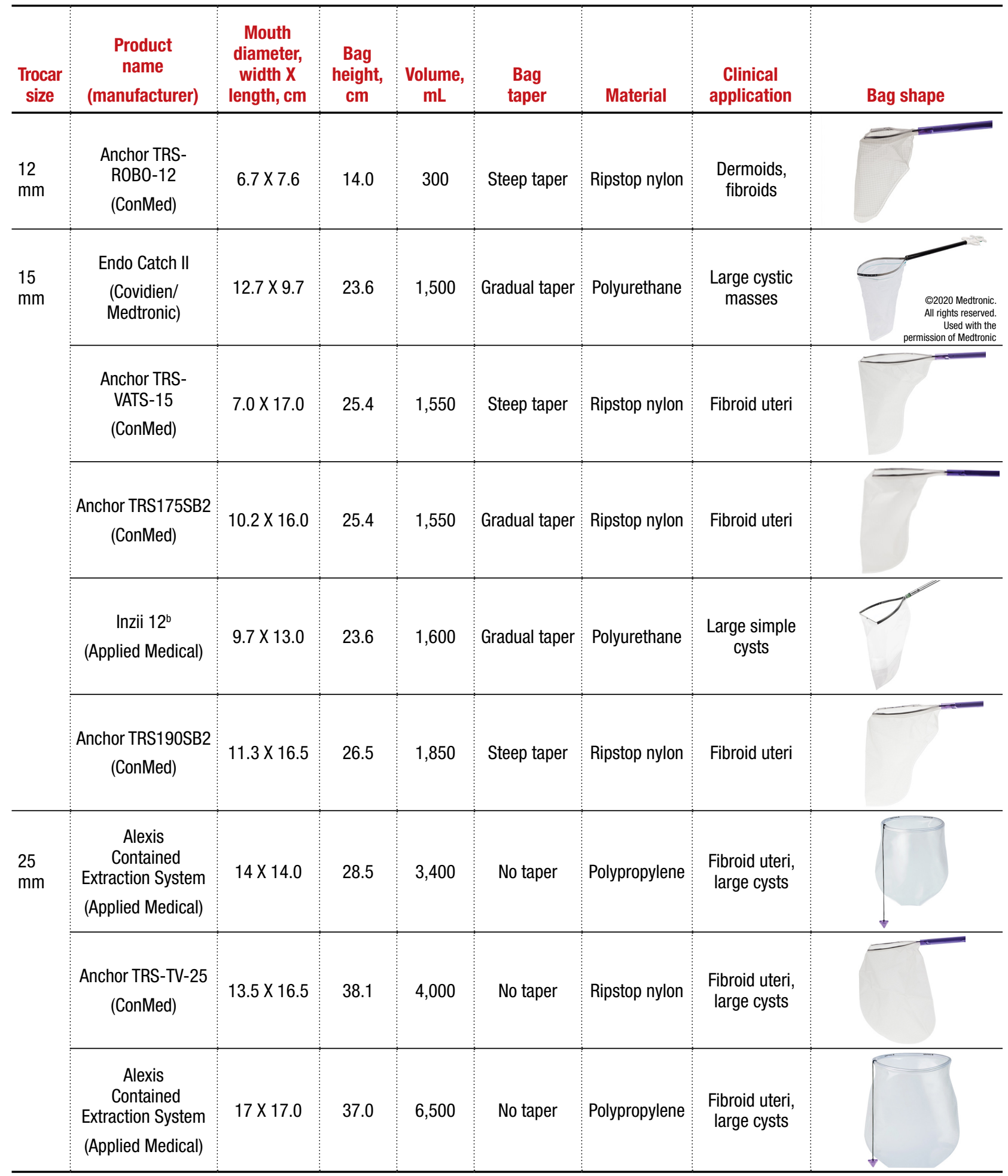

aThe Inzii 5-mm laparoscopic bag fits into the 5-mm Applied Medical Kii Access System trocar, which has a larger internal and outer diameter than a conventional 5-mm trocar. As such, this bag can also be inserted through a conventional 8-mm trocar, or requires removal of the 5-mm trocar prior to insertion of the bag directly through a 5-mm skin incision.

'The Inzii 12-mm laparoscopic bag fits into the 12-mm Applied Medical Kii Access System trocar, which has a larger internal and outer diameter than a conventional 12-mm trocar. As such, this bag can also be inserted through a conventional 15-mm trocar, or requires removal of the 12-mm trocar prior to insertion of the bag directly through a 12-mm skin incision. 
diameter, volume, bag shape, construction material, and best clinical application.

The following are terms used to refer to the components of a laparoscopic retrieval bag:

- Mouth diameter: diameter at the entrance of a fully opened bag (FIGURE 1)

- Bag volume: the total volume a bag can

\section{FIGURE 1 Laparoscopic retrieval device components and terminology}

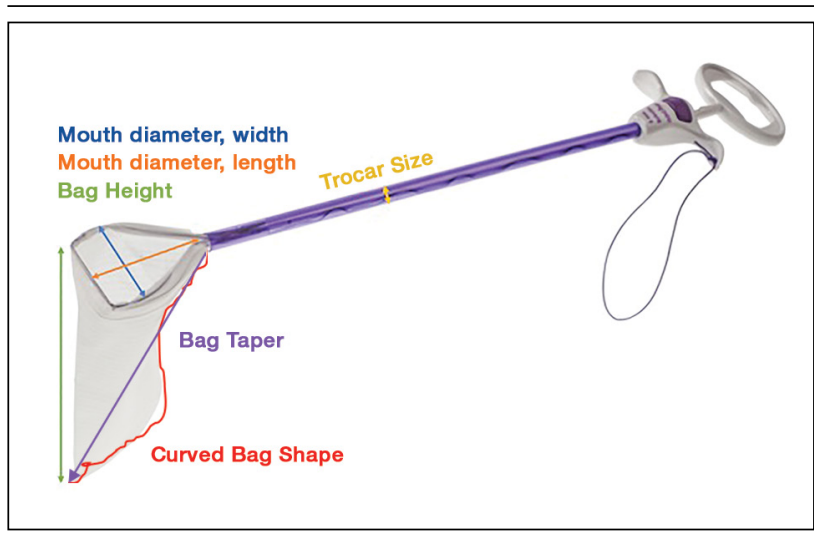

\section{FIGURE 2 Shapes of laparoscopic retrieval bags}

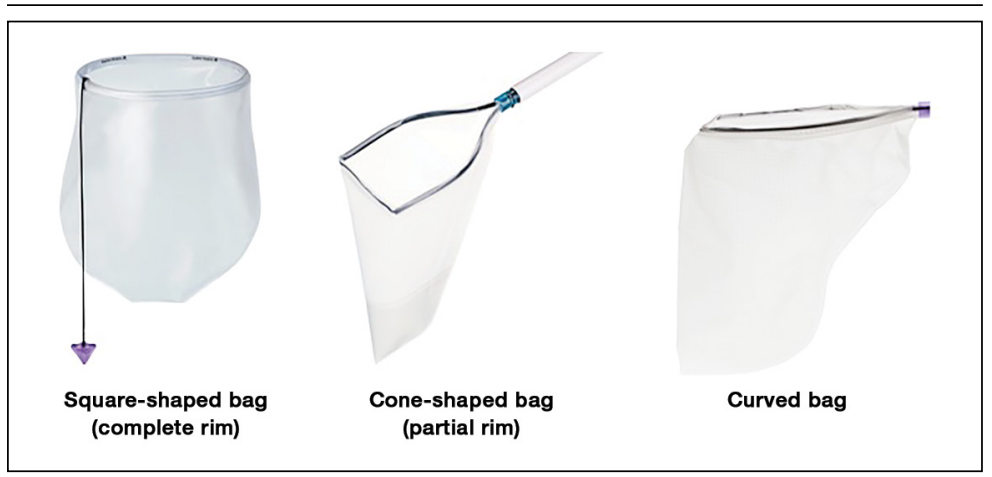

FIGURE 3 Taper configuration on laparoscopic retrieval bags

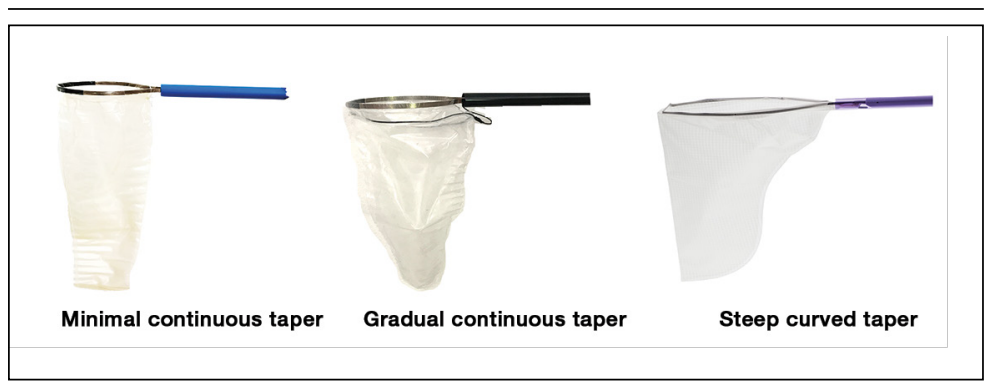

accommodate when completely full

- Bag rim: characteristics of the rim of the bag when opened (that is, rigid vs soft rim, complete vs partial rim mechanism to hold the bag open) (FIGURE 2)

- Bag shape: the shape of the bag when it is fully opened (square shaped vs cone shaped vs curved bag shape) (FIGURE 2)

- Bag taper (severity and type): extent the bag is tapered from the rim of the bag's entrance to the base of the bag; categorized by taper severity (minimal, gradual, or steep taper) and type (continuous taper or curved taper) (FIGURE 3)

- Ball fit: the maximum spherical specimen size that completely fits into a bag and allows it to cinch closed (FIGURE 4)

- Bag strength: durability of a bag when placed on tension during specimen extraction (weak, moderate, or extremely durable).

\section{Mouth diameter}

Bag manufacturers often differentiate bag sizes by indicating "volume" in milliliters. Bag volume, however, offers little clinical value to surgeons, as pelvic mass dimensions are usually measured in centimeters on imaging. Rather, an important characteristic for bag selection is the diameter of the rim of the bag when it is fully opened-the so-called bag mouth diameter. For a specimen to fit, the 2 dimensions of the specimen must be smaller than the dimensions of the bag entrance.

Notably, the number often linked to the specimen bag-as, for example, in the 10-mm Endo Catch bag (Covidien/Medtronic) describes the width of the shaft of the bag before it is opened rather than the mouth diameter of the opened bag. The number actually correlates with the trocar size necessary for bag insertion rather than with the specimen size that can fit into the bag. Therefore, a 10-mm Endo Catch bag cannot fit a $10-\mathrm{cm}$ mass, but rather requires a trocar size of $10 \mathrm{~mm}$ or greater for insertion of the bag. Fully opened, the mouth diameters of the $10-\mathrm{mm}$ Endo Catch bag are roughly $6 \mathrm{~cm} \times 7 \mathrm{~cm}$, which allows for delivery of a 6 - $\mathrm{cm}$ mass.

Because 2 bags that use the same trocar size for insertion may have vastly differing 


\section{FIGURE 4 Effects of laparoscopic retrieval bag taper and mouth diameter on ball fit}

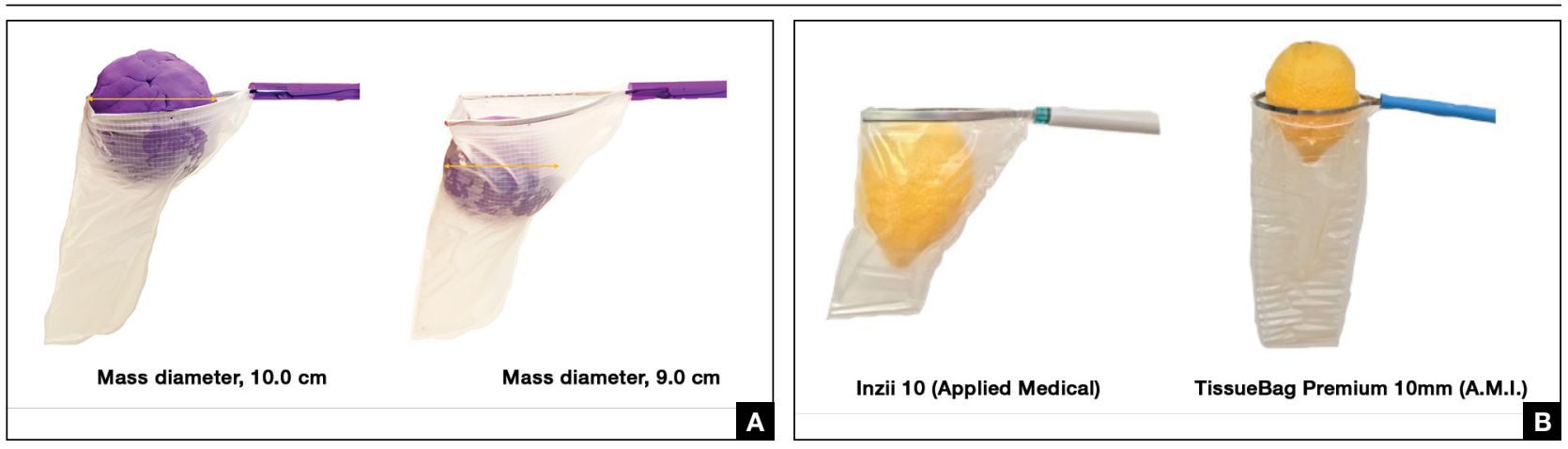

A Bag taper. Two examples of masses with different mass diameters within the same Anchor TRS175SB2 bag (ConMed). Left: The mass diameter of the specimen is just able to be accommodated by the mouth diameter of the bag, but the degree of bag taper hinders closure. The ball fit for the bag is smaller than the mouth diameters. Right: Although the specimen's mass diameter is smaller than the mouth diameter of the bag, the ball fit is appropriate, allowing for the bag to be cinched closed.

B Bag mouth diameter. Although these bags have the same trocar size, their mouth diameters are different, resulting in different ball fits. Left: The specimen fits nicely within the bag. Right: The specimen passes through the mouth diameter, but because of bag's taper it is unable to be placed within the bag.

bag dimensions, the surgeon must know the bag mouth diameters when selecting a bag to remove the presenting pathology. For example, the Inzii 12 (Applied Medical) laparoscopic bag has mouth diameters of $9.7 \mathrm{~cm} \times 13.0 \mathrm{~cm}$, whereas the Anchor TRSROBO-12 (ConMed) has mouth diameters of $6.7 \mathrm{~cm} \times 7.6 \mathrm{~cm}$ (TABLE). Although both bags can be inserted through a $12-\mathrm{mm}$ trocar, both bags cannot fit the same size mass for removal.

\section{Shape and taper}

Laparoscopic bags come in various shapes (curved, cone, or square shaped), with varying levels of bag taper (steep, gradual, or no taper) (FIGURES 2 and 3). While taper has little impact on long and skinny specimens, taper may hinder successful bagging of bulky or spherical specimens.

Each bag has different grades of taper regardless of mouth diameter or trocar size. For round masses, the steeper the taper, the smaller the mass that can comfortably fit within the bag. This concept is connected to the idea of "ball fit," explained below.

In addition, bag shape may affect what mass size can fit into the bag. An irregularly shaped curved bag or a bag with a steep taper may be well suited for removal of multiple specimens of varying sizes or soft masses that are malleable enough to conform to the bag's shape (such as a ruptured ovarian cyst). Alternatively, a square-shaped bag or a bag with minimal taper would better accommodate a round mass.

\section{Ball fit}

When thinking about large circular masses, such as myomas or ovarian cysts, one must consider the ball fit. This refers to the maximum spherical size of the specimen that fits completely within a bag while allowing the bag to cinch closed. Generally, this is an estimation that factors in the bag shape, extent of the bag taper, bag mouth diameter, and specimen shape and tissue type. At times, although a mass can fit through the bag's mouth diameter, a steep taper may prevent the mass from being fully bagged and limit closure of the bag (FIGURE 4).

Curved bags like the Anchor TRSVATS-15 (ConMed), which have a very narrow bottom, are prone to a limited ball fit, and thus the bag mouth diameter will not correlate with the largest mass size that can be fitted within the bag. Therefore, if using a steeply tapered bag for removal of large round masses, do not rely on the bag's mouth

\section{FAST \\ TRACK}

Ball fit refers to the maximum spherical size of the specimen that fits completely within a bag while allowing the bag to cinch closed 


\section{Laparoscopic specimen retrieval bags in gyn surgery: Expert guidance on selection}

FAST

TRACK

Trocar size

for bag selection

refers to the

minimum trocar

diameter needed

to insert the

laparoscopic bag diameter for bag selection. The surgeon must visualize the ball fit within the bag, taking into account the specimen size and shape, bag shape, and bag taper. In these scenarios, using the diameter of the midportion of the opened bag may better reflect the mass size that can fit into that bag.

\section{Bag strength}

Bag strength depends on the material used for bag construction. Most laparoscopic bags in the United States are made of 3 different materials: polyurethane, polypropylene, and ripstop nylon.

Polyurethane and polypropylene are synthetic plastic polymers; in bag form they are stretchy and, under extreme force, may tear. They are best used for bagging fluidfilled cysts or soft pliable masses that will not require extensive bag or tissue handling, such as extraction of large leiomyomas. Polyurethane and polypropylene bags are more susceptible to puncture with sharp laparoscopic instruments or scalpels, and care must be taken to avoid accidentally cutting the bag during tissue extraction.

Alternatively, bags made of ripstop nylon are favored for their bag strength. Ripstop nylon is a synthetic fabric that is woven together in a crosshatch pattern that makes it resistant to tearing and ripping. It was developed originally during World War II as a replacement for silk parachutes. Modern applications include its use in sails, kites, and high-quality camping equipment. This material has a favorable strength-to-weight ratio, and, in case of a tear, it is less prone to extension of the tear. For surgical applications, these bags are best used for bagging specimens that will require a lot of bag manipulation and tissue extraction. However, the ripstop fabric takes up more space in the incision than polyurethane or polypropylene, leaving the surgeon with less space for tissue extraction. Thus, as a tradeoff for bag strength, the surgeon may need to extend the incision a little, and a small self-retracting wound retractor may be necessary to allow visibility for safe tissue extraction when using a ripstop nylon bag compared with others.

\section{Trocar selection is important}

While considering bag selection, the surgeon also must consider trocar selection to allow for laparoscopic insertion of the bag. Trocar size for bag selection refers to the minimum trocar diameter needed to insert the laparoscopic bag. Most bags are designed to fit into a laparoscopic trocar or into the skin incision that previously housed the trocar. Trocar size does not directly correlate with bag mouth diameter; for example, a 10-mm laparoscopic bag that can be inserted through a 10or 12-mm trocar size cannot fit a $10-\mathrm{cm}$ mass (see the mouth diameter section above).

A tip to maximize operating room (OR) efficiency is to start off with a larger trocar, such as a $12-\mathrm{mm}$ trocar, if it is known that a laparoscopic bag with a 12-mm trocar size will be used, rather than starting with a $5-\mathrm{mm}$ trocar and upsizing the port site incision. This saves time and offers intraoperative flexibility, allowing for the use of larger instruments and quicker insufflation.

Furthermore, if the specimen has a solid component and tissue extraction is anticipated, consider starting off with a large trocar, one that is larger than the bag's trocar size since the incision likely will be extended. For example, even if a myoma will fit within a 10-mm laparoscopic bag made of ripstop nylon, using a $15-\mathrm{mm}$ trocar rather than a 10-mm trocar may be considered since the skin and fascial incisions will need to be extended to allow for cold-cut tissue extraction. Starting with the larger $15-\mathrm{mm}$ trocar may offer surgical advantages, such as direct needle delivery of larger needles for myometrial closure after myomectomy or direct removal of smaller myomas through the trocar to avoid bagging multiple specimens.

\section{Putting it all together}

To optimize efficiency in the OR for specimen removal, we recommend streamlining OR flow and reducing waste by first considering the specimen size, tissue type, bag shape, and trocar selection. Choose a bag by taking into account the bag mouth diameter and the amount of taper you will need to obtain an 


\section{Laparoscopic specimen retrieval bags in gyn surgery: Expert guidance on selection}

CONTINUED FROM PAGE 42

\section{$\overline{\text { FAST }}$}

TRACK

\section{Consider starting off with a larger} trocar rather than spending the time to upsize a trocar if you plan to use a large bag or intend to extend the trocar incision for a contained tissue extraction appropriate ball fit. If the tissue type is soft and pliable, consider a polyurethane or polypropylene bag and the smallest bag size possible, even if it has a narrow bag shape and taper.

However, if the tissue type is solid, the shape is round, and the mass is large (requiring extensive tissue extraction for removal), consider a bag made of ripstop nylon and factor in the bag shape as well as the bag taper. Using a bag without a steep taper may allow a better fit.

After choosing a laparoscopic bag, select the appropriate trocars necessary for completion of the surgery. Consider starting off with a larger trocar rather than spending the time to upsize a trocar if you plan to use a large bag or intend to extend the trocar incision for a contained tissue extraction. These tips will help optimize efficiency, reduce equipment wastage, and prevent intra-abdominal spillage.

Keep in mind that all procedures, including specimen removal using containment systems, have inherent risks. For example, visualization of the mass within the bag and visualization of vital structures may be hindered by bulkiness of the bag or specimen. There is also a risk of bag compromise and

References

1. Desai VB, Wright JD, Lin H, et al. Laparoscopic hysterectomy route, resource use, and outcomes: change after power morcellation warning. Obstet Gynecol. 2019;134:227-238.

2. American College of Obstetricians and Gynecologists. ACOG committee opinion No. 444: choosing the route of hysterectomy for benign disease. Obstet Gynecol. 2009;114:1156-1158.

3. Liu H, Lu D, Wang L, et al. Robotic surgery for benign gynecological disease. Cochrane Database Syst Rev. 2012;2:CD008978.

4. Wright JD, Herzog TJ, Tsui J, et al. Nationwide trends in the performance of inpatient hysterectomy in the United States. Obstet Gynecol. 2013;122(2 pt 1):233-241.

5. Turner LC, Shepherd JP, Wang L, et al. Hysterectomy surgery trends: a more accurate depiction of the last decade? Am J Obstet Gynecol. 2013;208:277.el-7.

6. Holme JB, Mortensen FV. A powder-free surgical glove bag for retraction of the gallbladder during laparoscopic cholecystectomy. Surg Laparosc Endosc Percutan Tech. 2005;15:209-211.

7. Siedhoff MT, Cohen SL. Tissue extraction techniques for leiomyomas and uteri during minimally invasive surgery. Obstet Gynecol. 2017;130:1251-1260.

8. US Food and Drug Administration. Laparoscopic uterine power morcellation in hysterectomy and myomectomy: FDA safety communication. April 17, 2014. https://wayback .archive-it.org/7993/20170722215731/https:/www.fda.gov /MedicalDevices/Safety/AlertsandNotices/ucm393576.htm. Accessed September 22, 2020 leakage, whether through manipulation of the bag or puncture during specimen extraction. Lastly, even though removing a specimen within a containment system minimizes spillage and reports of in-bag cold-knife tissue extraction in women with histologically proven endometrial cancer have suggested that it is safe, laparoscopic bags have not been proven to prevent the dissemination of malignant tissue fragments. ${ }^{16,17}$

Overall, the inherent risks of specimen extraction during minimally invasive surgery are far outweighed by the well-established advantages of laparoscopic surgery, which carries lower risks of surgical complications such as bleeding and infection, shorter hospital stay, and quicker recovery time compared to laparotomy. There is no doubt minimally invasive surgery offers many benefits.

In summary, for best bag selection, it is equally important to know the characteristics of the pathology as it is to know the features of the specimen retrieval systems available at your institution. Understanding both the pathology and the equipment available will allow the surgeon to make the best surgical decisions for the case.

9. AAGL. AAGL practice report: morcellation during uterine tissue extraction. J Minim Invasive Gynecol. 2014;21:517-530.

10. American College of Obstetricians and Gynecologists. ACOG committee opinion No. 770: uterine morcellation for presumed leiomyomas. Obstet Gynecol. 2019;133:e238-e248.

11. Society of Gynecologic Oncology website. SGO position statement: morcellation. December 1, 2013. https://www .sgo.org/newsroom/position-statements-2/morcellation/. Accessed September 22, 2020.

12. Advincula AP, Truong MD. ExCITE: minimally invasive tissue extraction made simple with simulation. OBG Manag. 2015;27(12):40-45

13. Solima E, Scagnelli G, Austoni V, et al. Vaginal uterine morcellation within a specimen containment system: a study of bag integrity. J Minim Invasive Gynecol. 2015;22:1244-1246.

14. Ghezzi F, Casarin J, De Francesco G, et al. Transvaginal contained tissue extraction after laparoscopic myomectomy: a cohort study. BJOG. 2018;125:367-373.

15. Dotson S, Landa A, Ehrisman J, et al. Safety and feasibility of contained uterine morcellation in women undergoing laparoscopic hysterectomy. Gynecol Oncol Res Pract. 2018;5:8.

16. Favero G, Miglino G, Köhler C, et al. Vaginal morcellation inside protective pouch: a safe strategy for uterine extration in cases of bulky endometrial cancers: operative and oncological safety of the method. J Minim Invasive Gynecol. 2015;22:938-943.

17. Montella F, Riboni F, Cosma S, et al. A safe method of vaginal longitudinal morcellation of bulky uterus with endometrial cancer in a bag at laparoscopy. Surg Endosc. 2014;28:1949-1953. 